\title{
Characterization of cosmic rays and direction dependence in the Polar Region up to $88 \mathrm{~km}$ altitude
}

\author{
Balázs Zábori*, Attila Hirn, Sándor Deme, István Apáthy, and Tamás Pázmándi \\ Radiation Protection Department, Centre for Energy Research, Hungarian Academy of Sciences, Budapest, Hungary \\ *Corresponding author: zabori.balazs@energia.mta.hu
}

Received 2 September 2015 / Accepted 26 January 2016

\begin{abstract}
Aims: The sounding rocket experiment REM-RED was developed to operate on board the REXUS-17 rocket in order to measure the intensity of cosmic rays. The experiment was launched from the ESRANGE Space Center $\left(68^{\circ} \mathrm{N}, 21^{\circ} \mathrm{E}\right)$ on the 17 th of March 2015 at the beginning of the most intense geomagnetic storm within the preceding 10 years. The experiment provided the opportunity to measure the intensity of cosmic rays in the Polar Region up to an altitude of $88 \mathrm{~km}$ above sea level.

Methods: The experiment employed Geiger-Müller (GM) counters oriented with their axes perpendicular to each other in order to measure the cosmic ray intensity during the flight of the rocket. This measurement setup allowed performing direction-sensitive measurements as well. During the ascent phase the rocket was spinning and hence stabilized along its longitudinal axis looking close to the zenith direction. This phase of the flight was used for studying the direction dependence of the charged particle component of the cosmic rays.

Results: In comparison with earlier, similar rocket experiments performed with GM tubes at lower geomagnetic latitudes, significantly higher cosmic radiation flux was measured above $50 \mathrm{~km}$. A non-isotropic behavior was found below $50 \mathrm{~km}$ and described in detail for the first time in the Polar Region. This behavior is in good agreement with the results of the TECHDOSE experiment that used the same type of GM tubes on board the BEXUS-14 stratospheric balloon.
\end{abstract}

Key words. Cosmic ray - Radiation Environment - Atmosphere - Space weather - Solar activity

\section{Introduction}

Among many other student projects, the European Space Agency (ESA) Education Office announces a call for proposals annually for the REXUS/BEXUS (Rocket and Balloon Experiments for University Students) flights for university students. The REXUS/BEXUS program allows students from universities and higher education colleges across Europe to carry out scientific and technological experiments on research rockets and balloons. ${ }^{1}$ Each year two sounding rockets (called REXUS) are launched $145 \mathrm{~km}$ north of the Arctic Circle in Sweden, carrying experiments designed and built by university student teams. The launch site is located at $68^{\circ} \mathrm{N}$ latitude and at $324 \mathrm{~m}$ above the sea level. The typical REXUS vehicle consists of a one-stage Improved Orion motor (M112 Hawk) and the payloads. Depending on the total experiment mass this rocket can reach a maximum altitude of $90 \mathrm{~km}^{2}$

The Hungarian student experiment REM-RED was selected to take part in the REXUS-17/18 launch campaign. The experiment flew on board the REXUS-17 sounding rocket. The experiment used Geiger-Müller (GM) tubes for the active monitoring of the cosmic ray intensity (charged particles and gamma photons). The main technical and scientific support was given to the experiment team by the Centre for Energy Research, Hungarian Academy of Sciences (MTA EK). The measurement setup, first introduced in the TECHDOSE stratospheric balloon experiment (Zábori et al. 2013, 2015a, 2015b),

\footnotetext{
${ }^{1} \mathrm{http}: / / w w w . r e x u s b e x u s . n e t$

2 EuroLaunch, REXUS User Manual, RX_UserManual_v7-13_15, Dec. 14, 2014.
}

provided the opportunity to characterize the direction dependence of the cosmic ray intensity in the atmosphere up to the maximum altitude of the REXUS-17, approximately $88 \mathrm{~km}$ above sea level.

REXUS-17 was launched on the 17th of March 2015 at 8:15 UTC from the ESRANGE Space Center at the beginning of the most intense geomagnetic storm within the preceding 10 years.

\section{Radiation environment during the flight of the REXUS-17 rocket}

Generally the incoming primary cosmic rays interact with the Earth's magnetosphere and the atmosphere providing a complex radiation environment (Schaefer 1959). Cosmic ray fluxes vary with geomagnetic latitude and solar activity. Protons, with about $85 \%$ contribution, are the main constituent of cosmic rays at the Earth's orbit and they dominate the production of secondary particles (Schaefer 1959; Grieder 2001). The charged particle and photon flux in the atmosphere shows a local maximum in the altitude range of about 15-26 km, called Pfotzer Maximum (Pfotzer 1936), which corresponds to the maximum of the secondary particle production. The Pfotzer Maximum shows a significant change with the geomagnetic latitude (Bazilevskaya et al. 2008). In the Polar Region, from where the REXUS-17 rocket was launched, the expected value is within the range between 20 and $26 \mathrm{~km}$.

The directional behavior of cosmic rays at ground level is well studied; however, no scientific data about the directionality 
Table 1. The main technical parameters of the GM tubes used.

\begin{tabular}{lcc}
\hline \hline \multicolumn{1}{c}{ GM tube type } & ZP1200 & ZP1210 \\
\cline { 3 - 4 } \cline { 3 - 4 } \multicolumn{1}{c}{ Supplier } & Centronic & Centronic \\
\hline Number of GM tubes in the experiment & 2 & 4 \\
Sensitive length (mm) & 15 & 140 \\
Sensitive diameter (mm) & 6 & 20 \\
Sensitive cross-area of one tube in the lateral direction $\left(\mathrm{cm}^{2}\right)$ & $10^{-3}-10^{2}$ & 28 \\
Gamma dose rate range (mGy h ${ }^{-1}$ ) & 28 & $3 \times 10^{-4}-10^{1}$ \\
Count rate at $10^{-2} \mathrm{mGy} \mathrm{h}^{-1}$ using Cs-137 source (sensitivity) & 500 & 110 \\
Operating voltage (V) & & 500 \\
Photon sensitivity ratio (ZP1200/ZP1210) & & 0.25 \\
\hline
\end{tabular}

of the cosmic rays at higher altitudes up to approximately $200 \mathrm{~km}$ is available. Muons are the most significant component of the cosmic rays on the ground. Most of them are produced higher in the atmosphere, at around the Pfotzer Maximum and reach the ground from the top (Grieder 2001). The angular distribution of cosmic ray muons can be described with a $\cos ^{2} \theta$ ( $\theta$ is measured from the zenith) function, which means that the zenith direction has an overall dominancy over the horizontal directions at ground level (Grieder 2001).

The influence of solar activity on the cosmic ray flux has to be also taken into account. During higher solar activity periods the galactic cosmic ray flux decreases. Rocket experiments performed in the past showed a difference between solar quiet and solar active periods, which could be identified also at altitudes where the REXUS-17 flew (Shafer 1963).

The magnetic field of the Earth influences the radiation environment through the geomagnetic shielding effect of the magnetosphere. At high latitudes, where the magnetic cut-off rigidity $R_{c}$ is below $2 \mathrm{GV}$, the cut-off rigidity decreases significantly under disturbed geomagnetic conditions (Smart \& Shea 2005). Nominally the cut-off rigidity at the ESRANGE base is of the order of $0.1 \mathrm{GV}$ in the case of quiet geomagnetic conditions $\left(K p=2\right.$; Smart et al. 2006). ${ }^{3}$

According to NOAA, during the period between the 15th and the 19th of March 2015, the solar activity increased and some regions of the Sun produced solar flares. The first event was detected on the 16th at 10:58 UTC followed by a second one on the 17th at 22:34 UTC. During the rest of the week several C-class solar events were observed by the GOES satellites. ${ }^{4}$ In the energy range of $1-30 \mathrm{MeV}$, an increase in the solar proton flux was detected. In the case of the alpha particles, a three order of magnitude increase was observed from the middle of 15th of March until the end of 18th of March in the energy range of $1-20 \mathrm{MeV}$. The electron flux above $2 \mathrm{MeV}$ showed significant disturbances. Following the significant decrease of the geomagnetic cut-off rigidities the solar energetic particles may have reached the flight altitude range of the REXUS-17 rocket in the Polar Region; however taking into account the energy threshold limits imposed by the shielding of the rocket materials (see Table 2) it can be concluded that the solar energetic particles were not measured by the experiment directly, only the galactic cosmic rays were observed.

\footnotetext{
${ }^{3}$ Equivalent to $5 \mathrm{MeV}$ proton or $1 \mathrm{MeV}$ alpha particles' cut-off energy.

${ }^{4}$ An overview of the GOES-R program was given by M. Greg at the 94th Annual AMS Meeting and 10th Annual Symposium on Future National Operational Environmental Satellite Systems, February 4, 2014. Title: An Overview of the GOES-R Program (http://www.goes-r.gov/downloads/AMS/2014/pres/02-04/03-GregMandt.pdf).
}

\section{Methods and experiment description}

The REM-RED experiment included two different types of GM tubes: four pieces of type ZP1210 Centronic tubes with high sensitivity for measuring purposes and two pieces of type ZP1200 Centronic tubes with lower sensitivity for testing purposes. The higher sensitivity ZP1210 type GM tubes were duplicated for both orientations in order to decrease the statistical uncertainty of the measurements. Although the sensitivity in the case of the ZP1200 is very low, due to its small dimensions and low weight (length: $50 \mathrm{~mm}$, mass: $8 \mathrm{~g}$ ), this type might be preferable for space applications. Table 1 summarizes the main technical parameters of the GM tubes used. Besides the GM tubes, the REM-RED experiment employed accelerometers and several temperature, current, and voltage sensors for housekeeping purposes.

Figure 1 shows the mechanical assembly, especially the location and the orientation of the GM tubes inside the experiment. At least two specimens of each GM tube type were used and placed perpendicular to each other in order to provide direction dependent measurements.

According to the theory of GM tubes an incoming photon produces secondary electrons (mostly Compton electrons) predominantly in the wall of the GM tube, which are counted by the tube. Therefore, in the case of photons the directional sensitivity of GM tubes is close to uniform in $4 \pi$. Charged particles moving along a straight line cross directly the sensitive volume of the GM tube and produce ionization mostly inside this volume. Therefore the entrance window for a charged particle moving on a straight line is the sensitive cross-area perpendicular to the velocity vector of the charged particle.

Based on the geometry of the GM tubes the angular sensitivity can be calculated both for the vertically and the horizontally oriented tubes. Figure 2 indicates that the horizontally oriented GM tubes are much more sensitive to the radiation coming from the zenith than the vertically oriented ones due to the different effective surfaces. However, direction dependent measurements are possible only in the case of the charged particles, since in the case of photons the angular distribution is almost uniform.

For general characterization of the cosmic ray environment measurement data provided by the four ZP1210 type GM tubes were used with a total effective area of $86.8 \mathrm{~cm}^{2}$. The total effective aluminum equivalent thickness of the shielding for particles coming from the horizontal plane was $2 \mathrm{~g} \mathrm{~cm}^{-2}$ and for particles coming from the zenith direction about $4 \mathrm{~g} \mathrm{~cm}^{-2}$ aluminum equivalent. The difference between the effective thicknesses is due to the mechanical structure of 

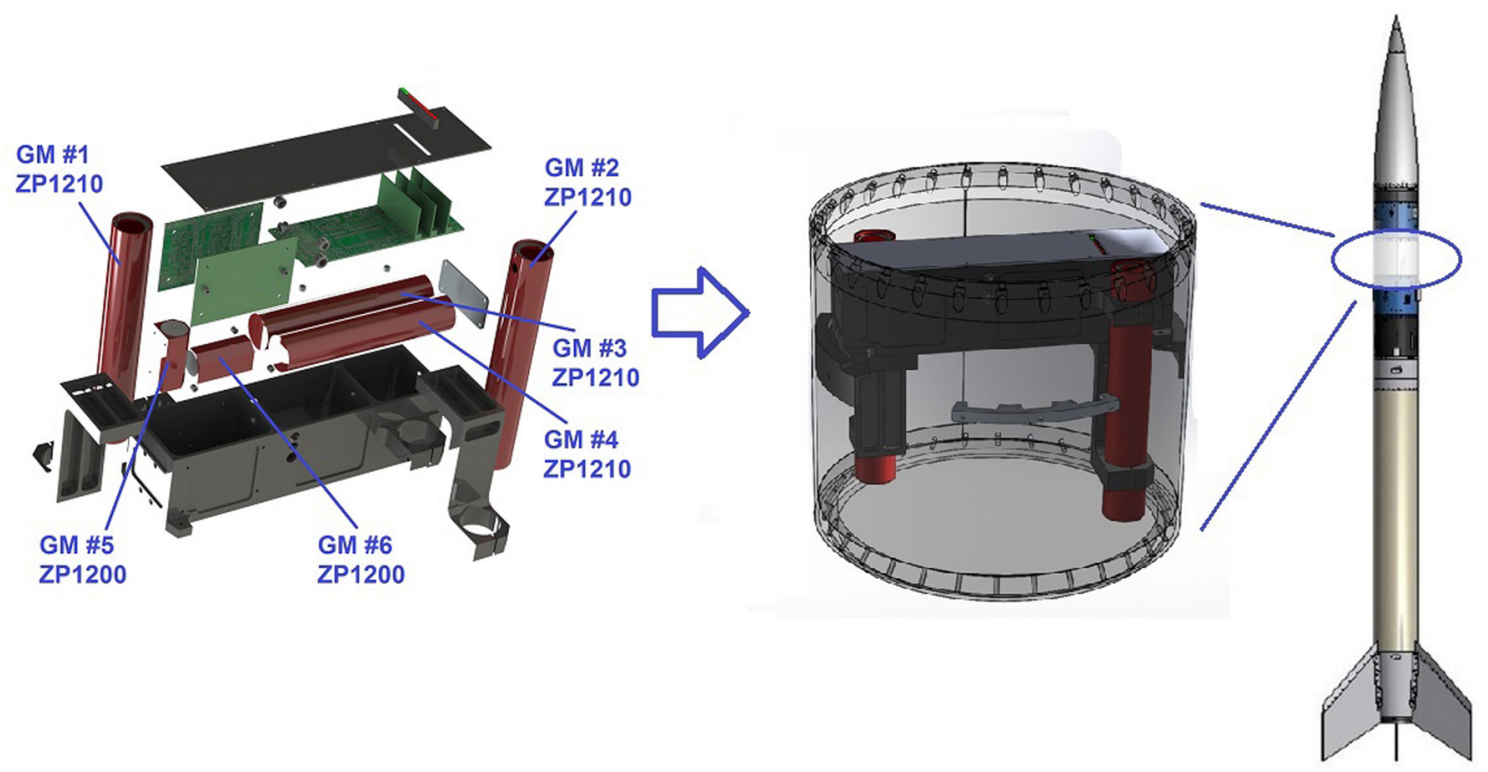

Fig. 1. Exploded view of the REM-RED experiment and its location on board the REXUS-17 sounding rocket.

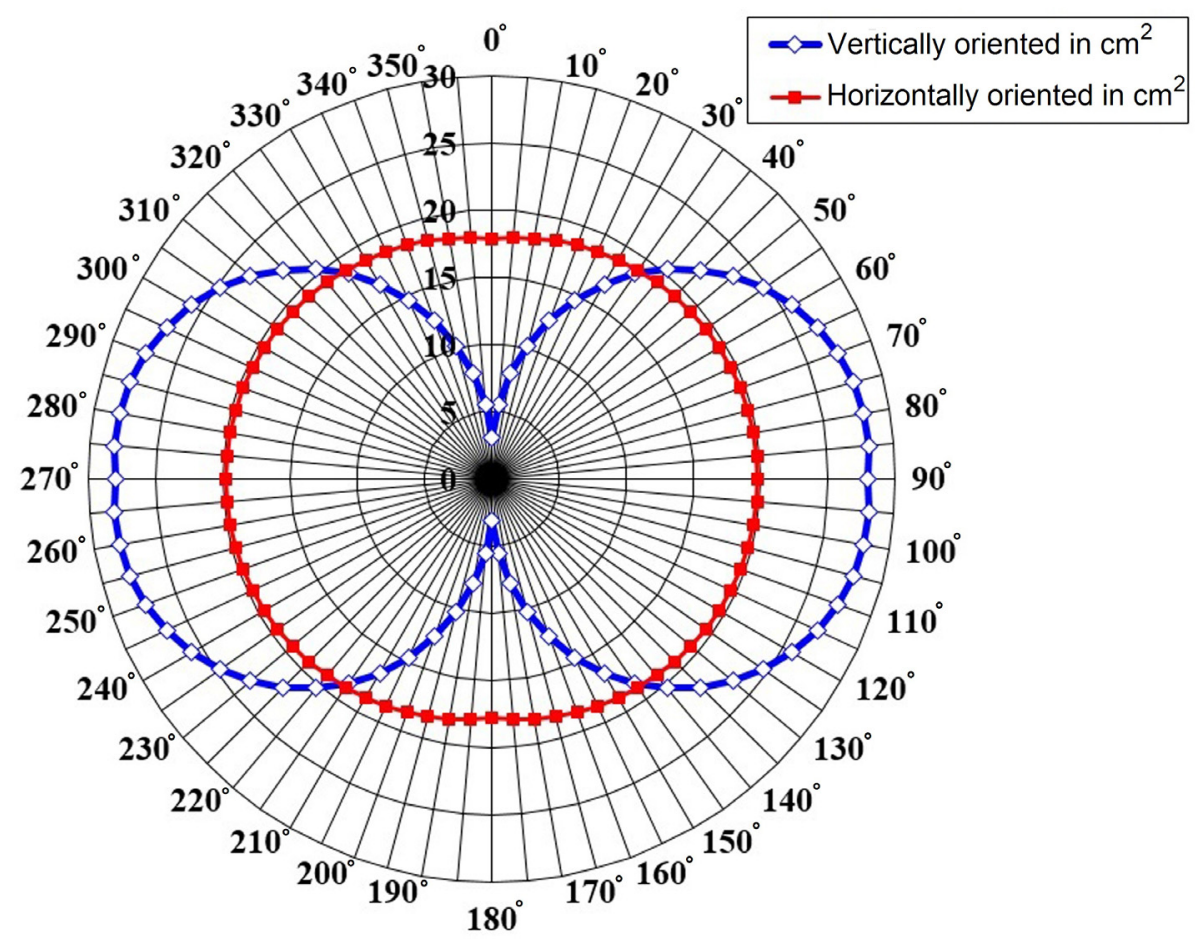

Fig. 2. The effective surface of the vertically and horizontally oriented ZP1210 type GM tubes as a function of zenith angle assuming a $2 \pi$ isotropic angular particle distribution from the upper half space.

Table 2. The threshold energies of the REM-RED GM measurements.

\begin{tabular}{ccc}
\hline \hline Component & \multicolumn{2}{c}{ Lowest energy threshold } \\
\cline { 2 - 3 } & $\begin{array}{c}2 \mathrm{~g} \mathrm{~cm}^{-2} \mathrm{Al} \\
\text { equivalent shielding }\end{array}$ & $\begin{array}{c}4 \mathrm{~g} \mathrm{~cm}^{-2} \mathrm{Al} \\
\text { equivalent shielding }\end{array}$ \\
\hline Photons* $(\mathrm{keV})$ & 10 & 25 \\
Protons $(\mathrm{MeV})$ & 20 & 50 \\
Alpha particles & 130 & 200 \\
$(\mathrm{MeV})$ & & \\
\hline
\end{tabular}

* Corresponding to 10th-value thickness. the rocket since above the REM-RED another experiment module was located. The lowest energy thresholds for some cosmic ray components that can reach the detectors are summarized in Table 2.

According to Table 2 it can be assumed that the largest contribution comes from gamma photons or high-energy charged particles (mainly galactic cosmic rays), which means that the difference in the shielding thickness can be almost neglected. The corresponding cut-off energies are much lower than these values, which means that the geomagnetic environment or the disturbances had no significant influence on the measured results. 


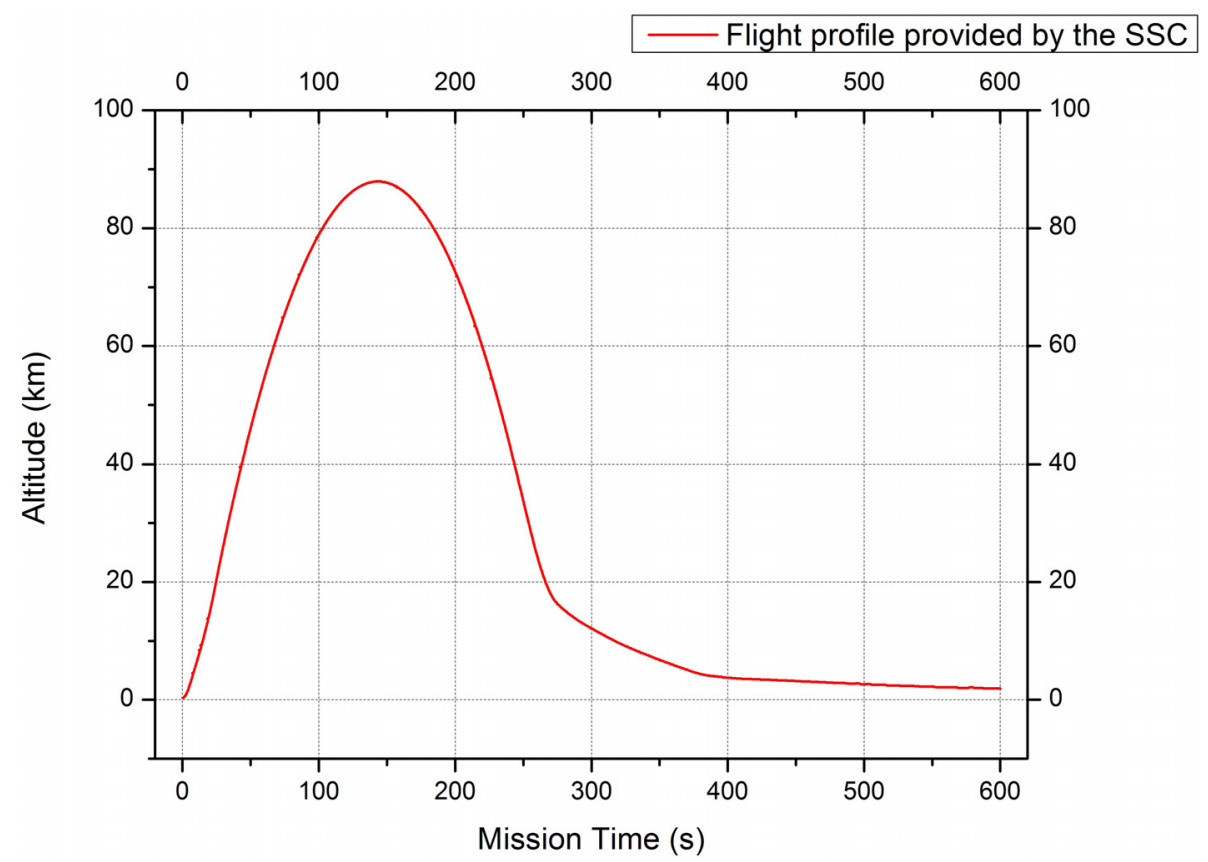

Fig. 3. The flight profile of the REXUS-17 sounding rocket (data provided by the Swedish Space Corporation (SSC)).

Table 3. The average count rates measured with the REM-RED GM tubes before the launch.

\begin{tabular}{|c|c|c|c|c|}
\hline \multirow{2}{*}{$\begin{array}{c}\text { GM tube type } \\
\text { Orientation }\end{array}$} & \multicolumn{2}{|c|}{ ZP1210 } & \multicolumn{2}{|c|}{ ZP1200 } \\
\hline & Horizontal & Vertical & Horizontal & Vertical \\
\hline No. of GM tubes & 2 & 2 & 1 & 1 \\
\hline Count rate on ground $\left(\mathrm{s}^{-1} ; 324 \mathrm{~m}\right.$ above sea level) & $1.30 \pm 0.07$ & $1.08 \pm 0.07$ & $0.23 \pm 0.02$ & $0.18 \pm 0.02$ \\
\hline Total number of counts & 1324 & 1095 & 116 & 91 \\
\hline Horizontal/vertical count rate ratio & \multicolumn{2}{|c|}{$1.21 \pm 0.05$} & \multicolumn{2}{|c|}{$1.27 \pm 0.18$} \\
\hline
\end{tabular}

\section{Results}

The total flight time of REXUS-17 was about $10 \mathrm{~min}$ from its launch until its landing. The apogee was reached $144 \mathrm{~s}$ after the lift-off at an altitude of $88 \mathrm{~km}$ (see the flight profile in Fig. 3). The GM units of the REM-RED experiment were switched on approximately $10 \mathrm{~min}$ before the launch and they were switched off $300 \mathrm{~s}$ later to prevent them from being damaged during the last phase of the reentry. The motor burned out and separated $26 \mathrm{~s}$ after the lift-off. The rocket was spinning around its central axis with an average rate of $4 \mathrm{~Hz}$. The central axis of the rocket was oriented for the lift-off $7^{\circ}$ from the zenith direction and it was stable until the apogee was reached. The rocket landed $46 \mathrm{~km}$ away from ESRANGE.

The flight performance of the experiment was 100\%; almost all data were received by telecommunication during the flight. The missing frames were recovered after the experiment had been shipped back to the ESRANGE base and the content of the on-board memory had been downloaded.

The time resolution of the GM-counter data was $1 \mathrm{~s}$ during the flight, which means better altitude resolution at the top of the trajectory and during the landing, when the velocity of the rocket was lower (the altitude resolution in the worst case was $1.0 \mathrm{~km}$ ) than during the ascent phase. After lift-off the orientation of the rocket was stable and looking almost into the zenith direction until it reached the apogee. This phase of the flight was used for direction dependent measurements (see Sect. 4.2). During the reentry phase the rocket was no more stabilized. However, its velocity was much lower; thus, this phase was used for non-directional characterization of the radiation environment with better statistics regarding altitude resolution.

\subsection{The qualification of the cosmic ray flux in the Polar Region up to $88 \mathrm{~km}$ altitude}

Before the launch of the rocket the REM-RED experiment was operated on the surface in its launch position to obtain radiation results on the surface with good statistics. The average count rates for the horizontal and vertical GM tubes can be found in Table 3. At around sea level, mostly muons, which have well-known angular distribution (Grieder 2001), can be detected. Our pre-flight measurement results shown in Table 3 are in line with the expectations.

Figure 4 shows the count rates measured with the higher sensitivity tubes. The peak count rate $\left(\sim 60 \mathrm{~s}^{-1}\right)$ was reached at $23.6 \pm 1.0 \mathrm{~km}$. In the case of the ZP1200 measurement results, the region above $50 \mathrm{~km}$ was also evaluated and provided an average count rate of $9.21 \pm 0.16 \mathrm{~s}^{-1}$ (obtained from two ZP1200 GM tube measurements).

\subsection{The direction dependence of the cosmic radiation flux in the Polar Region up to $88 \mathrm{~km}$ altitude}

Our first study on a possible directional dependence of the cosmic radiation flux as a function of altitude was performed in 


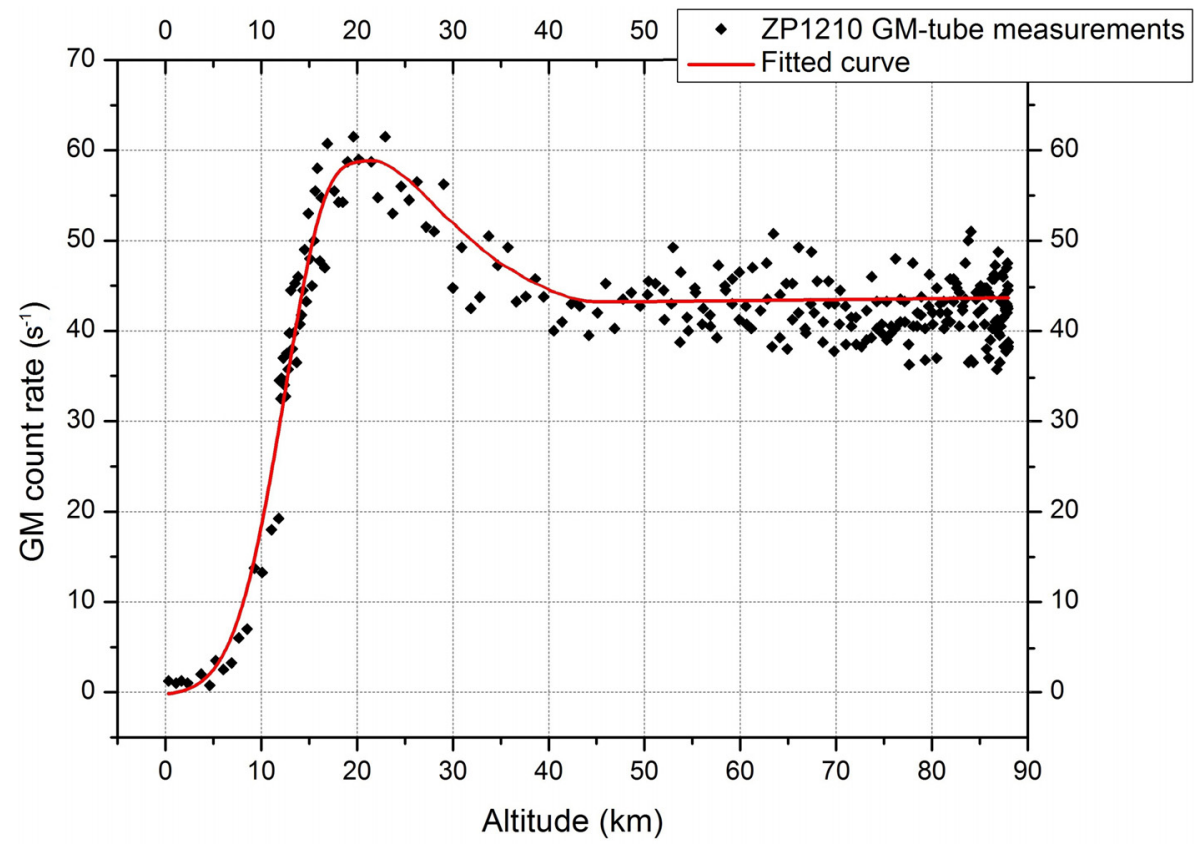

Fig. 4. Count rates measured with the ZP1210 GM tubes as a function of flight altitude during the flight of the REXUS-17 rocket (for better visibility only every fifth data point is plotted).

2012 with a BEXUS (Balloon Experiments for University Students) experiment called TECHDOSE. TECHDOSE flew on board the BEXUS-14 balloon, which was launched on the 24th of September 2012 from the same launch area (ESRANGE) as the REXUS-17 rocket (Zábori et al. 2013, 2015a, 2015b). The experiment used among several other radiation instruments the same ZP1210 type GM tubes as the REM-RED sounding rocket experiment. To monitor the directionality of the radiation environment a similar arrangement was used as in the case of the REXUS flight. The maximum floating altitude of the balloon was $28.6 \mathrm{~km}$. The measured GM profile can be seen in Figure 5 (Zábori et al. 2015b). Below the altitude range of the Pfotzer Maximum the profile is very similar to the one measured two years later during the REXUS-17 flight. In the case of the balloon experiment, the effective shielding in front of the GM tubes was the same, however the results showed directional dependence. This implies that the direction dependent profiles in the case of the rocket flight cannot be the consequence of the different shielding and thus the different levels of the secondary particle production in the shielding material. In the case of the BEXUS flight, the uncertainties of the measured GM data were relatively low since $60 \mathrm{~s}$ integration time was used due to the relatively low ascent velocity of the balloon (the maximum velocity was $3.3 \mathrm{~m} \mathrm{~s}^{-1}$ ). This meant $\sim 2-3 \%$ relative error in the count rates above $10 \mathrm{~km}$ altitude.

A similar but less significant direction dependence has been already measured by Adams et al. (2011) using GM tubes in balloon flights up to $25 \mathrm{~km}$ altitude in the frame of BASE (Balloon Assisted Stratospheric Experiments). In their rocket experiments, Shafer \& Yarygin (1961) used a perpendicular GM tube arrangement similar to that of TECHDOSE and REM-RED; however, no direction dependent data were reported.

BEXUS-14 and REXUS-17 flew at the same geomagnetic latitude and the TECHDOSE and REM-RED experiments employed the same type of GM tubes in a similar geometry.
This provided the possibility to extend the directional radiation data measured by TECHDOSE with the REM-RED experiment up to $88 \mathrm{~km}$ altitude. Figure 6 shows the direction dependence of the measured count rates during the REXUS-17 rocket flight.

The results of the REXUS-17 REM-RED are shown in Figure 6. All curves were smoothed over five data points. We could measure similar directionality with the ZP1200 type GM tubes as with the ZP1210 type GM tubes having higher sensitivity. However, these data cannot be used for further data evaluation due to the large uncertainties. Above $15 \mathrm{~km}$ altitude $5 \mathrm{~s}$ integration time was used. In order to have better statistics, the ZP1210 type GM tubes were duplicated in each direction providing double sensitive cross-area to detect the cosmic radiation. The statistical uncertainties of the measured radiation data above $15 \mathrm{~km}$ were $\sim 4-5 \%$, which was sufficient to draw conclusions about the directionality of the cosmic radiation flux above the Pfotzer Maximum. Since below $15 \mathrm{~km}$, the uncertainties in the measured data were larger than $5 \%$, these data points were not included in the data evaluation.

\section{Discussion}

The maximum count rates measured with the REM-RED experiment were found at $23.6 \pm 1.0 \mathrm{~km}$ altitude in agreement with earlier reports (Bazilevskaya et al. 2008). The shape of the measured GM tube count rate profile as a function of altitude agrees well with the measurements performed by Van Allen in 1947-1948 using V-2 and Aerobee rockets at lower latitudes $\left(41^{\circ} \mathrm{N}\right)$ (Van Allen \& Tatel 1948; Gangnes et al. 1949). In 1958 geophysical rocket measurements conducted by Shafer \& Yarygin (1961) provided a very similar profile for the altitude dependence of the count rates. Above $50 \mathrm{~km}$ the expected plateau was found with an average count rate of $42.28 \pm 0.35 \mathrm{~s}^{-1}$ obtained from four ZP1210 GM tube measurements. 


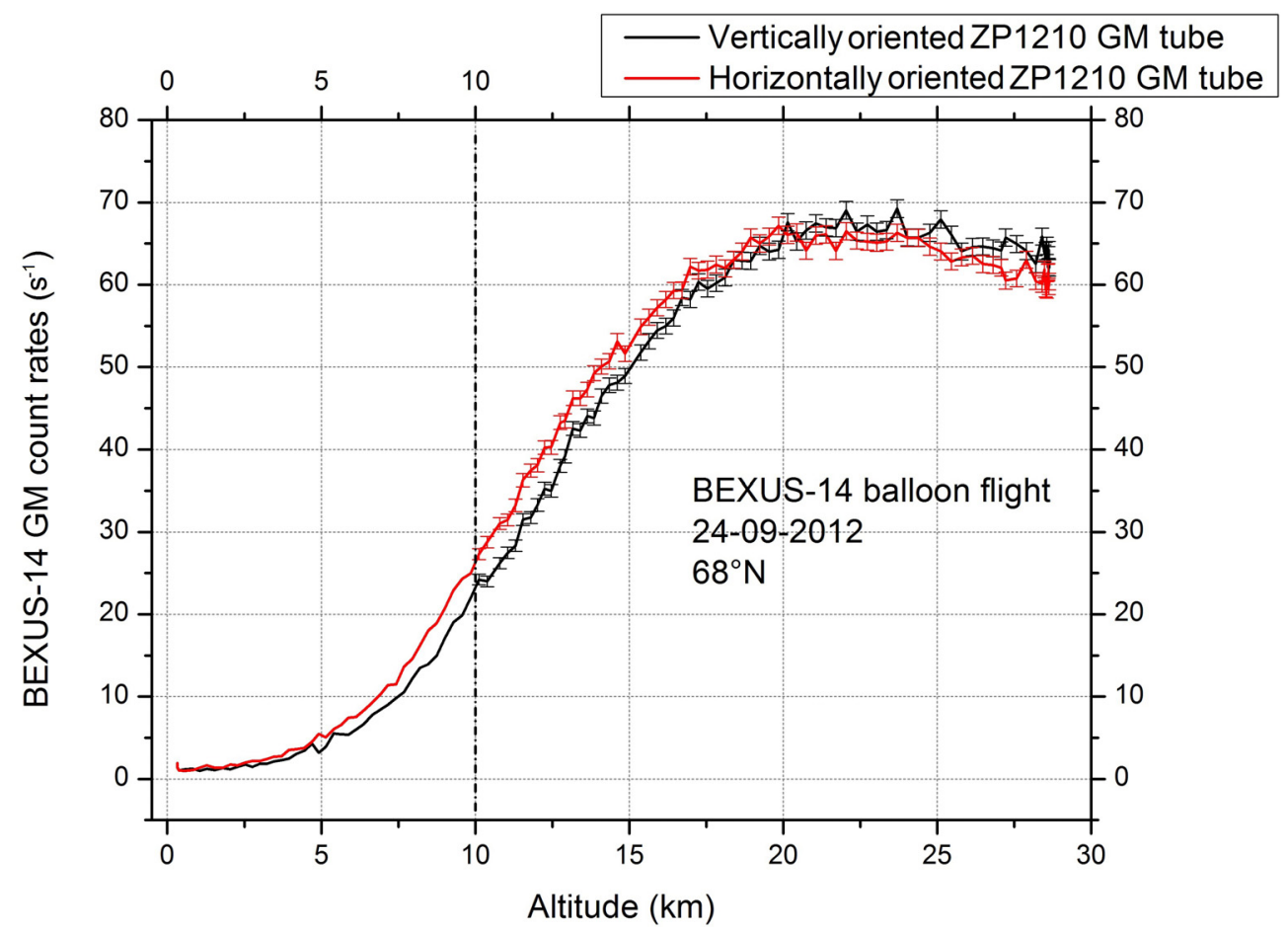

Fig. 5. The direction dependence of the count rates up to $28.6 \mathrm{~km}$ altitude measured by ZP1210 GM tubes oriented horizontally and vertically in the BEXUS-14 stratospheric balloon flight. The dashed line indicates $10 \mathrm{~km}$ altitude, above this altitude the uncertainties of the ZP1210 values are $2-3 \%$ (Zábori et al. 2015b).

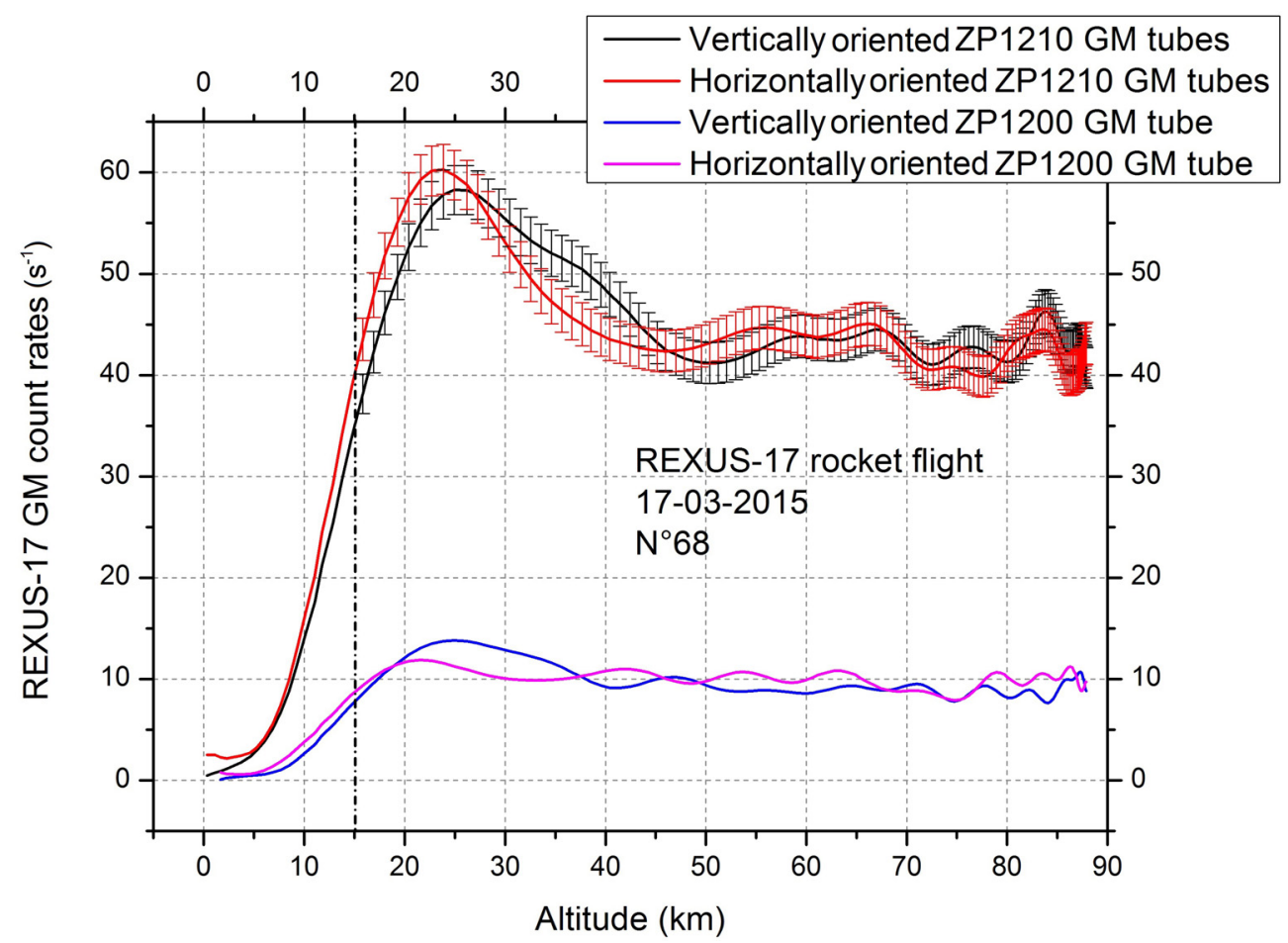

Fig. 6. The direction dependence of the count rates measured by ZP1210 and ZP1200 type GM tubes in the REXUS-17 REM-RED experiment. The uncertainties of the measured data estimated from $5 \mathrm{~s}$ integration time are indicated in the ZP1210 curves above $15 \mathrm{~km}$ with vertical error bars. Only this region was used to draw conclusions about the directionality of the cosmic radiation flux. The dashed line indicates the $15 \mathrm{~km}$ altitude; above this altitude the uncertainties of the ZP1210 values are 4-5\%. Note that for the data evaluation the duplicated ZP1210 GM tubes were used with duplicated sensitive volume normalized to the sensitive volume of one single tube in order to have better comparison with the BEXUS GM profiles in Figure 5. 
Table 4. The altitude corresponding to the Pfotzer Maximum based on BEXUS-14 data.

\begin{tabular}{l}
\hline \hline For particles coming predominantly from the zenith direction \\
$\begin{array}{l}\text { For particles coming predominantly from the horizontal directions } \\
\text { The uncertainty of the altitude determination of the Pfotzer Maximum is inferred from the error of the Gaussian function fit to the measured } \\
\text { data, the vertical component of the maximum ascent velocity of the balloon, and the integration time of the GM tubes. }\end{array}$ \\
\hline
\end{tabular}

There is a well-known relationship in an isotropic flux distribution between the count rate $N$ of a GM tube, the geometric factor $G$, and the flux of the particles $j$ :

$$
N=G \cdot j=0.5 \cdot \pi^{2} \cdot a \cdot l \cdot(1+a /(2 \cdot l)) \cdot j,
$$

where $l$ is the sensitive length, $a$ is the sensitive diameter of the GM tube, and $j$ is the particle flux (Van Allen \& Tatel 1948). Using this equation for the data measured in the plateau region and neglecting the spurious background of the GM tubes, the measured particle flux was found to be $0.245 \pm 0.005 \mathrm{~cm}^{-2} \mathrm{~s}^{-1} \mathrm{sr}^{-1}$. It is significantly higher than the $0.120 \pm 0.009 \mathrm{~cm}^{-2} \mathrm{~s}^{-1} \mathrm{sr}^{-1}$ flux measured by Van Allen \& Tatel (1948) using the same formula as given above. This can be attributed to the different geomagnetic latitudes and thus to the difference in the cut-off rigidities. The flight by Van Allen was carried out at significantly lower geomagnetic latitude $\left(41^{\circ} \mathrm{N}\right)$ where the cut-off energy reduced the flux of charged particles to a much greater extent. Additionally it has to be noted that (1) is valid only in the case of an isotropic flux; however, the calculations were made only for the GM plateau regions, where the flux was observed to be isotropic in the upper half space, thus the radiation flux can be considered isotropic.

According to the GM tube results obtained in the frame of the BEXUS-14 (10-28.6 km altitude range) and the REXUS17 (15-88 km altitude range) flights, the directional behavior of the cosmic radiation flux in the Polar Region could be described up to an altitude of $88 \mathrm{~km}$. Below the altitude corresponding to the Pfotzer Maximum, the radiation coming from the zenith direction is dominant $(+15 \%$ difference $)$. This behavior changes above the altitude of the Pfotzer Maximum ( $-10 \%$ difference). The inferred direction dependence originates mainly from charged particles as the GM tubes have significant directional sensitivity only for the charged particles. Above $50 \mathrm{~km}$ the radiation intensity is almost constant and no significant direction dependence could be observed up to $88 \mathrm{~km}$.

The altitude of the measured Pfotzer Maximum shows direction dependence as well. In the case of charged particles coming from the zenith direction the Pfotzer Maximum is about $1 \mathrm{~km}$ lower than for particles coming from the horizontal plane (Table 4). This phenomenon could be seen in the measured data during the BEXUS-14 and the REXUS-17 flight as well; however in the case of the REM-RED experiment, the altitude determination of the Pfotzer Maximum had much larger uncertainty due to the higher velocity of the REXUS rocket. In Figure 4 the contribution from both the vertically and the horizontally oriented ZP1210 GM tubes appears to result in a widened peak in the plot due to the different Pfotzer Maximum for the two orientations.

The REXUS rocket and the BEXUS balloon experiments were carried out at the same high latitude location in the Polar Region. The major difference was the uncertainty of the measured radiation data as a function of altitude, since the vertical velocity of the rocket was much higher than that of the balloon.

\section{Conclusion}

The BEXUS-14 TECHDOSE stratospheric balloon and the REXUS-17 REM-RED sounding rocket cosmic ray experiments flew at a latitude of $68^{\circ} \mathrm{N}$ in the Polar Region and up to an altitude of $28.6 \mathrm{~km}$ in the case of the balloon and $88 \mathrm{~km}$ in the case of the rocket experiment in order to measure the cosmic ray environment using GM tubes of different sensitivities.

During the ascent phase the rocket was stabilized in its launch orientation looking almost into the zenith direction. This phase of the flight was used to evaluate the direction dependence of the cosmic radiation flux. A significant direction dependence was found and characterized in more detail for the first time in the Polar Region up to $88 \mathrm{~km}$. Below the altitude of the Pfotzer Maximum the radiation coming from the zenith direction is dominant, which changes above the Pfotzer Maximum until the radiation intensity stabilizes at about $50 \mathrm{~km}$ altitude. Above $50 \mathrm{~km}$ no significant direction dependence could be observed. Considering a $2 \pi$ uniform particle distribution from the upper half space and using the same GM particle flux calculation method which was introduced by Van Allen, the detected particle flux above $50 \mathrm{~km}$ in the rocket experiment was found to be $0.245 \pm 0.005 \mathrm{~cm}^{-2} \mathrm{~s}^{-1} \mathrm{sr}^{-1}$.

Below $50 \mathrm{~km}$ it can be assumed that the cosmic radiation flux is non-isotropic mostly due to the charged particle component. The results obtained during the BEXUS-14 balloon flight using the same type of GM tubes showed the same direction dependence as the rocket experiment up to the maximum altitude of the balloon. Additionally it was found that the altitude of the Pfotzer Maximum shows direction dependence as well. In the case of charged particles coming from the zenith direction, the altitude of the Pfotzer Maximum was $1 \mathrm{~km}$ lower than for particles coming from the horizontal directions.

In the future, it will be worth to study the directional behavior of the cosmic ray flux in the atmosphere and the behavior of the Pfotzer Maximum itself in more detail by performing similar experiments at similar geomagnetic latitudes.

Acknowledgements. The authors acknowledge the services provided by the organizers of the REXUS/BEXUS, especially the European Space Agency Educational Office, the German Aerospace Center (DLR), the Swedish Space Corporation (SSC), the Swedish National Space Board (SNSB), and the experts of the space organizations who supported the student teams throughout the project. The authors wish to thank the work of the REM-RED team members and the scientific and engineering support provided by the experts of the Centre for Energy Research, Hungarian Academy of Sciences. The authors wish to thank also for the support provided by the BL Electronics Limited and the BOSCH Lifetime Research and Testing Laboratory as well as the help in the literature survey provided by Rémy Paixao from the École des Mines de Nantes on GM tube measurements on board rocket and balloon flights performed in the past. The general REXUS-17 flight data were provided by the Swedish Space Corporation. The space weather related data used in this paper are available at the National Oceanic and Atmospheric Administration Space Weather Prediction Center webpage. 
The REXUS REM-RED experiment was co-funded in the frame of the PECS (Plan for European Cooperating States) Contract No. 4000111902/14/NL/NDe.

The editor thanks Daniel Heynderickx and an anonymous referee for their assistance in evaluating this paper.

\section{References}

Adams, A., E. Brauer, and J. Stroman. A small Geiger counter array. Proceedings of the 2nd Annual Academic High-Altitude Conference (AHAC), Iowa State University, Ames, USA, 2011.

Bazilevskaya, G.A., I.G. Usoskin, E.O. Flückiger, R.G. Harrison, L. Desorgher, et al. Cosmic ray induced ion production in the atmosphere. Space Sci. Rev., 137, 149-173, 2008, DOI: $10.1007 / \mathrm{s} 11214-008-9339-\mathrm{y}$.

Gangnes, A.V., J.F. Jenkins, Jr., and J.A. Van Allen. The cosmic-ray intensity above the atmosphere. Phys. Rev., 75, 57, 1949, DOI: 10.1103/PhysRev.75.57.

Grieder, P.K.F. Cosmic Rays at Earth: Researcher's Reference Manual and Data Book, Elsevier Science B. V, The Netherlands, ISBN: 0-444-50710-8, 2001.

Pfotzer, G. Dreifachkoinzidenzen der Ultrastrahlung aus vertikaler Richtung in der Stratosphare. Physikalische Zeitschift, 102, 23 40, 1936.

Schaefer, H.J. Radiation and man in space. In: Advances in space science, Vol. 1, edited by F., Ordway. Academic Press, New York - London, pp. 267-339, 1959.

Shafer, Yu.G., and A.V. Yarygin. Measurements of cosmic rays on geophysical rockets. Planet. Space Sci., 8, 165-172, 1961, DOI: 10.1016/0032-0633(61)90261-6.
Shafer, Yu.G. Effect of drop in solar activity on intensity of cosmic rays according to sounding rocket measurements in 1958 and 1960. Planet. Space Sci., 11, 427-430, 1963, DOI: 10.1016/0032-0633(63)90269-1.

Smart, D.F., and M.A. Shea. A review of geomagnetic cutoff rigidities for earth-orbiting spacecraft. Adv. Space Res., 36, 2012-2020, 2005, DOI: 10.1016/j.asr.2004.09.015.

Smart, D.F., M.A. Shea, A.J. Tylka, and P.R. Boberg. A geomagnetic cutoff rigidity interpolation tool: Accuracy verification and application to space weather. Adv. Space Res., 37, 1206-1217, 2006, DOI: 10.1016/j.asr.2006.02.011.

Van Allen, J.A., and H.E. Tatel. The cosmic-ray counting rate of a single Geiger counter from ground level to 161 kilometers altitude. Phys. Rev., 73, 245, 1948.

Zábori, B., I. Apáthy, A. Csőke, S. Deme, A. Hirn, T. Hurtony, J. Pálfalvi, and T. Pázmándi. CoCoRAD and TECHDOSE cosmic radiation experiments on board BEXUS stratospheric research balloons, Proceedings of the 21st ESA Symposium on European Rocket and Balloon Programmes and Related Research (ESA SP721, October 2013), ISBN: 978-92-9092-285-8, pp. 307-314, 2013.

Zábori, B., A. Gerecs, Gy.Á. Hurtonyné, D. Bényei, F. Náczi, and T. Hurtony. REM-RED cosmic radiation experiment on-board the REXUS-17 sounding rocket. Proceedings of the 22nd ESA Symposium on European Rocket and Balloon Programmes and Related Research (ESA SP-730, September 2015), ISSN 1609042X, pp. 539-546, 2015a.

Zábori, B., A. Hirn, S. Deme, I. Apáthy, A. Csőke, T. Pázmándi, and P. Szántó. Space dosimetry measurements in the stratosphere using different active and passive dosimetry systems, Radiat. Prot. Dosim., 2015b, pp. 1-10, DOI: 10.1093/rpd/ncv442.

Cite this article as: Zábori B, Hirn A, Deme S, Apáthy I \& Pázmándi T. Characterization of cosmic rays and direction dependence in the Polar Region up to $88 \mathrm{~km}$ altitude. J. Space Weather Space Clim., 6, A12, 2016, DOI: 10.1051/swsc/2016006. 